\title{
Classificação social dos animais em Kaingáng
}

\author{
Aryon Dall'Igna Rodrigues
}

A organização social do povo Kaingáng tem como um de seus elementos básicos a divisão em metades exogâmicas e a subdivisão de cada uma destas em dois ou mais subgrupos. Segundo Nimuendajú ([1914] 1987:122), essa divisão se estendia a toda a natureza. Baldus (1937), entretanto, com base em seu trabalho de campo junto aos Kaingáng de Palmas, PR, concluiu que tal extensão não existia. Já Veiga (1994), cuja pesquisa básica foi com os Kaingáng de Xapecó, SC, confirma a informação de Nimuendajú. Durante meu trabalho de campo linguístico entre os Kaingáng de Mangueirinha, PR, que falam o mesmo dialeto que os de Palmas e os de Xapecó (dialeto central), um dos membros mais idosos da comunidade me voluntariou a classificação de grande número de espécies animais segundo as duas metades sociais e seus subgrupos. Como até agora, que eu saiba, nenhum levantamento dessa classificação foi publicado, apresento aqui os dados por mim obtidos, juntamente com algumas outras informações então registradas e referentes à classificação social dos Kaingáng.

Meu trabalho de campo em Mangueirinha foi em 1951. Eu tinha completado no ano anterior a licenciatura em Letras Clássicas na Universidade (Federal) do Paraná e viajei para lá, por conta própria, depois de ter obtido informações na 7a. Inspetoria do Serviço de Proteção aos Índios, em Curitiba. Tive como companheiro de viagem o Oldemar Blasi, que tinha completado sua licenciatura cm Geografia e História e que pretendia estrear-se em observações etnográficas, assim como eu ia estrear-me em documentação de uma língua indígena. $\mathrm{O}$ posto indígena era administrado como uma fazenda pelo encarregado do SPI, sr. Atílio Mazalotti, que foi muito simpático e cooperativo, tendo-me permitido mesmo consultar a documentação administrativa do posto, consulta que me estarreceu ao verificar que o Ministério da Agricultura, ao qual estava então subordinado o SPI, exigia relatórios minuciosos e com testemunhas sobre a morte de vacas ou cavalos, mas não pedia nada mais que a data do óbito nos casos de mortes de índios. 
Há 50 anos não havia no Brasil nenhuma possibilidade de aprender a fazer trabalho de campo linguístico. Tudo dependia de intuição, de bom senso, de observação crítica dos acertos e erros. Foi aquela primeira experiência aventureira em Mangueirinha que me fez querer sair do Brasil para aprender fonética prática, procedimentos de análise linguística e muito mais além do que tinha podido me passar o grande pioneiro que foi o professor Mansur Guérios, ele necessariamente mais autodidata do que eu, que tive a sorte de ser seu aluno desde o ginásio até a faculdade. Assim mesmo, registrei muitos dados do dialeto Kaingáng falado em Mangueirinha, na Campina do Kretã, tanto vocabulário como frases e alguns pequenos textos. Tudo registrado diretamente de ouvido, pois ainda não havia gravadores de som portáteis. Transcrição fonética em parte à maneira dos romanistas, em parte à maneira dos registros de Curt Nimuendajú (que usava basicamente o alfabeto standard de Lepsius).

Sobre o povo Kaingáng eu já tinha lido o que havia escrito outro pioneiro paranaense, Telêmaco Borba, em seu importante livro de 1908, Atualidade Indígena, e o que registrou o etnólogo Herbert Baldus em seus Ensaios de Etnologia Brasileira, de 937. Sobre a língua, além dos dados registrados por Borba, havia o ensaio de gramática e o dicionário de Frei Mansueto Barcatta de Val Floriana, publicados em 1918 e 1920 e, bem mais recentes, os Estudos sobre a língua caingangue de Mansur Guérios, publicados em 1942. Ainda não conhecia o que havia escrito Nimuendajú sobre esse povo na parte final de seu famoso trabalho sobre a religião dos Guaraní, de que só tomei conhecimento muito tardiamente, quando obtive uma fotocópia da tradução para o espanhol publicada no Peru por Juergen Riester em 1978.

Numa tarde conversava eu com José Luís dos Santos, conhecido como o velho Coelho, na varanda da casa do encarregado. Já tínhamos conversado sobre a divisão do povo em duas metades exogâmicas e a subdivisão de cada metade em dois grupos: numa das metades Kadnjerú e Votoro, na outra Kamée Wéjnyký (de que tratara Baldus). Em certo momento uma borboleta atravessou a varanda e eu perguntei como se dizia "borboleta". O velho Coelho respondeu: toto, e acrescentou: "Essa aí é votoro." Então eu lhe perguntei se os animais também se dividiam como os homens e ele disse que sim, que os bichos se dividiram na festa deles. Acrescentou ainda que o sol é kamé e que a lua é kadnjerú. Mas, quando perguntei se as plantas também se dividem assim, respondeu que não. Aí fui perguntando por vários animais e ele foi dizendo qual o grupo de cada um. Foram ao todo pouco mais de cem espécies. Ele não hesitava ao informar o grupo a que pertencia o animal, mas, sendo tantos nomes, eu quis verificar se, por acaso, não estaria ele classificando aleatoriamente. Por isso, em outro dia voltei a perguntar por vários dos mesmos animais, mas as respostas foram quase $100 \%$ consistentes com as anteriores; apenas para duas aves, a baitaca e a andorinha, houve indicação dupla, mas da mesma metade: kamé e wéjnyký 
e para uma só, o marreco, atribuição às duas metades: votoro e wéjnyký. Esses raros casos de discordância em indagações separadas no tempo fazem crer que o velho Coelho estava dando um conhecimento bem estabelecido. Em favor disso contam também dois casos (morcego e besouro) em que Coelho declarou não saber classificar: "Não esteve no fandango; não sei dividi ele."

Na lista anexa apresento os nomes dos animais em Português e em Kaingáng, seguido cada um pela indicação de seu grupo social. Estes grupos são indicados pelas seguintes abreviações: $\mathrm{Kr}=$ Kadnjerú, $\mathrm{Vt}=$ Votoro, $\mathrm{Km}$ = Kamé, $\mathrm{Wn}$ = Wéjnyký. Apresento os nomes não na sequência em que os pedi, a qual foi bastante aleatória, mas ordenados segundo categorias maiores: símios, quadrúpedes, aves, répteis, peixes, insetos. Para os nomes em Kaingáng dou a transcrição fonética que na época pude fazer, mas transliterada para os símbolos do Alfabeto Fonético Internacional (IPA) e seguida, sempre que possível, pela escrita ortográfica (ortografia na versão preliminar de maio de 1999 de uma nova edição do dicionário de Wiesemann). Escrevo em ortografia também, entre colchetes, alguns nomes compostos, de que só os componentes figuram no dicionário. Entre parênteses acrescento as traduções do dicionário que diferem em algum detalhe da que foi obtida de meu informante.

Quanto às borboletas, o velho Coelho disse que "uma é $k a^{\prime} m \varepsilon$ outra vo'toro, outra kadnje'ru, outra wejni'ki que morreu: é o espírito deles que virou borboleta, conforme o desenho da asa"; to'to tj 'borboleta azul' é "índio bom, não batizado"; to'to ku'fõ 'borboleta vermelha' "é um índio não batizado muito ruim, que morreu"; to'to ku'pri 'borboleta branca' "é um espírito dum homem

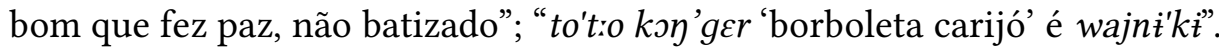

Embora sejam mencionados na literatura sobre os Kaingáng outros grupos ou "clãs", Coelho não fez referência senão aos quatro referidos acima, que são os mesmos mencionados pelo informante de Baldus no toldo das Lontras no município de Palmas. Apesar de coincidir em boa parte com as informações obtidas em outras áreas do povo Kaingáng, transcrevo aqui as minhas notas registradas na Campina do Kretã:

"Divisão da sociedade - grupos de casamento. A sociedade divide-se em duas metades, cada uma constituída por dois grupos; tanto as metades como os grupos são exogâmicos. Uma das metades é constituída pelos grupos kadnje'ru (kane'ru) e vo'toro, a outra pelos grupos ka'me e wejnit'ki (wæjniki, wajni'ki

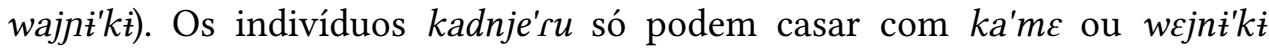
igualmente os vo'toro, os ka'me e wejni'ki só podem casar com kadnje'ru e vo'toro. A diferença dos grupos se manifesta por ocasião do casamento e por ocasião da festa dos mortos. Nesta, os índios pintam o rosto, servindo-se cada um do desenho característico do seu grupo. Segundo o índio João Luís dos Santos (kaje'ru, ca. 82 anos, nome do mato Je:r), a pintura dos kadnje'ru consiste em três pontos, dispostos em triângulo, repetidos em ambas as faces 
e na testa; a dos vo'toro é constituída por um pequeno círculo, feito com uma taquara cortada diametralmente, aplicado também sobre as faces e a testa; a dos ka'me consiste em duas linhas verticais paralelas, dispostas igualmente sobre as faces e a testa; e a dos $w \varepsilon j n \dot{t} k \dot{t}$ consiste em ângulos que, em ambas as faces, têm uma linha partindo do canto da boca, mais ou menos horizontal, e a outra em sentido mais ou menos vertical, com o vértice no meio da face; na testa é repetido o ângulo.

"Os filhos pertencem sempre ao grupo do pai; assim, todos os filhos e filhas do informante são kadnje'ru. Os kadnje'ru e os ka'me são mais numerosos que os vo'toro e os wejni'ki; e os ka'me são mais numerosos que os kadnje'ru.

"Segundo Se:r, os animais também se dividem como os homens nesses quatro grupos: a divisão foi feita na festa deles. O sol é $k a ' m \varepsilon$, a lua kadnje'ru: a lua é jam'bre do sol; é mulher do sol. As plantas não se dividem assim.”

A última informação, referente às plantas, não está de acordo com as informações obtidas por Nimuendajú em 1909, no alto rio do Peixe, SP, nem por Veiga em Xapecó, SC (Veiga 1994:60-61).

\section{REFERÊNCIAS}

BALDUS, H. Ensaios de etnologia brasileira. São Paulo: Companhia Editora Nacional, 1937.

BARCATTA DE VAL FLORIANA, M. Ensaio de grammatica Kainjgang. Revista do Museu Paulista, São Paulo, v. 10, p. 529-563, 1918.

Diccionarios Kainjgang-Portuguez e Portuguez-Kainjgang. Revista do Museu Paulista, São Paulo, v. 12, p. 1, p. 1-392, 1920.

BORBA, T. M. Actualidade indigena. Curitiba: Impressora Paranaense, 1908.

GUÉRIOS, R. F. M. Estudos sobre a língua caingangue. Notas histórico-comparativas. Arquivos do Museu Paranaense, Curitiba, v. 2, p. 97-177, 1942.

NIMUENDAJÚ-UNKEL, C. ([1914] 1978). Los mitos de la creación y destrucción del mundo como fundamentos de la religión de los Apapokuva-Guaraní. Tradução editada por Juergen Riester. Lima: Centro Amazónico de Antropología y Aplicación Prática.

As lendas da criação e destruição do mundo como fundamentos da região dos Apapokuva-Guarani. Trad. de C. Emmerich e E. B. Viveiros de Castro. São Paulo: HUCITEC/EDUSP.

VEIGA, J. Organização social e cosmovisão Kaingáng: uma introdução ao parentesco, casamento e nominação em uma sociedade jê meridional. 1994. Dissertação (Mestrado) - UNICAMP, Campinas.

WIESEMANN, U. Dicionário Kanhgág-Português-Kanhgág. Versão preliminar. |S. 1.|: Publicações Kaingáng só para uso particular, 1971. 
Lista de nomes de animais em Kaingáng de Mangueirinha, PR (dialeto central), em registro fonético, seguidos da escrita ortográfica (quando disponível) e da respectiva classificação social dos animais, apresentada em 1952 por José Luís ("Coelho") dos Santos, índio kadnierú, então com idade estimada de 82 anos. Registrada por Aryon D. Rodrigues.

\section{QUADRÚPEDES}

\begin{tabular}{|c|c|c|}
\hline anta & o'jor & ójor \\
\hline capivara & krig'ndig & krygnyg \\
\hline coelho & $\mathrm{ka}_{\sqrt{1} \text { Jinnbi'tõ }}$ & $\begin{array}{l}\text { [kasin my tũ } \\
\text { 'rato sem rabo'] }\end{array}$ \\
\hline cutia & ki'ðəg & kysóg \\
\hline guará & ho: & \\
\hline lontra & fog'f $\varepsilon j$ & fógfénh \\
\hline morcego & klik'fej & krygfẻj $j$ \\
\hline onça, tigre & mĩn & $n \tilde{u} g$ \\
\hline paca & kri's' & kryrã \\
\hline porco do mato & kre:y & krág \\
\hline porco espinho & krej'гег & \\
\hline puma, leão & miku'fõ & $\begin{array}{l}\text { [nũg kusũg } \\
\text { 'onça vermelha] }\end{array}$ \\
\hline quati & $\int \mathrm{e}:$ & xe \\
\hline raposa, gambá & dærko'kre & nér kókré \\
\hline serelepe & jati'ti & jótiti \\
\hline tateto & ok' $\int \tilde{\varepsilon}$ & ógs ã \\
\hline tatu do rabo duro & fæ'ñ̃j & fẽnẽnh \\
\hline
\end{tabular}




$\begin{array}{llll}\text { tatu do rabo mole } & \text { hì:y } & \text { hinh ('tatu') } & \mathrm{Kr} \\ \text { tatu mulinha } & \text { fæ'nẽj } \int \tilde{1} & \text { [fẽnẽnh sĩ] } & \mathrm{Km} \\ \text { veado } & \text { kع'mbe } & \text { kãme } & \mathrm{Kr}\end{array}$

\section{SÍMIOS}

\begin{tabular}{|c|c|c|}
\hline bugio branco & $\varepsilon g^{\prime} ? \mathrm{c}$ & ég'e ('bugio preto') \\
\hline bugio vermelho & goy, yəg & góg \\
\hline mico & kaj'nعг & kajẽr \\
\hline
\end{tabular}

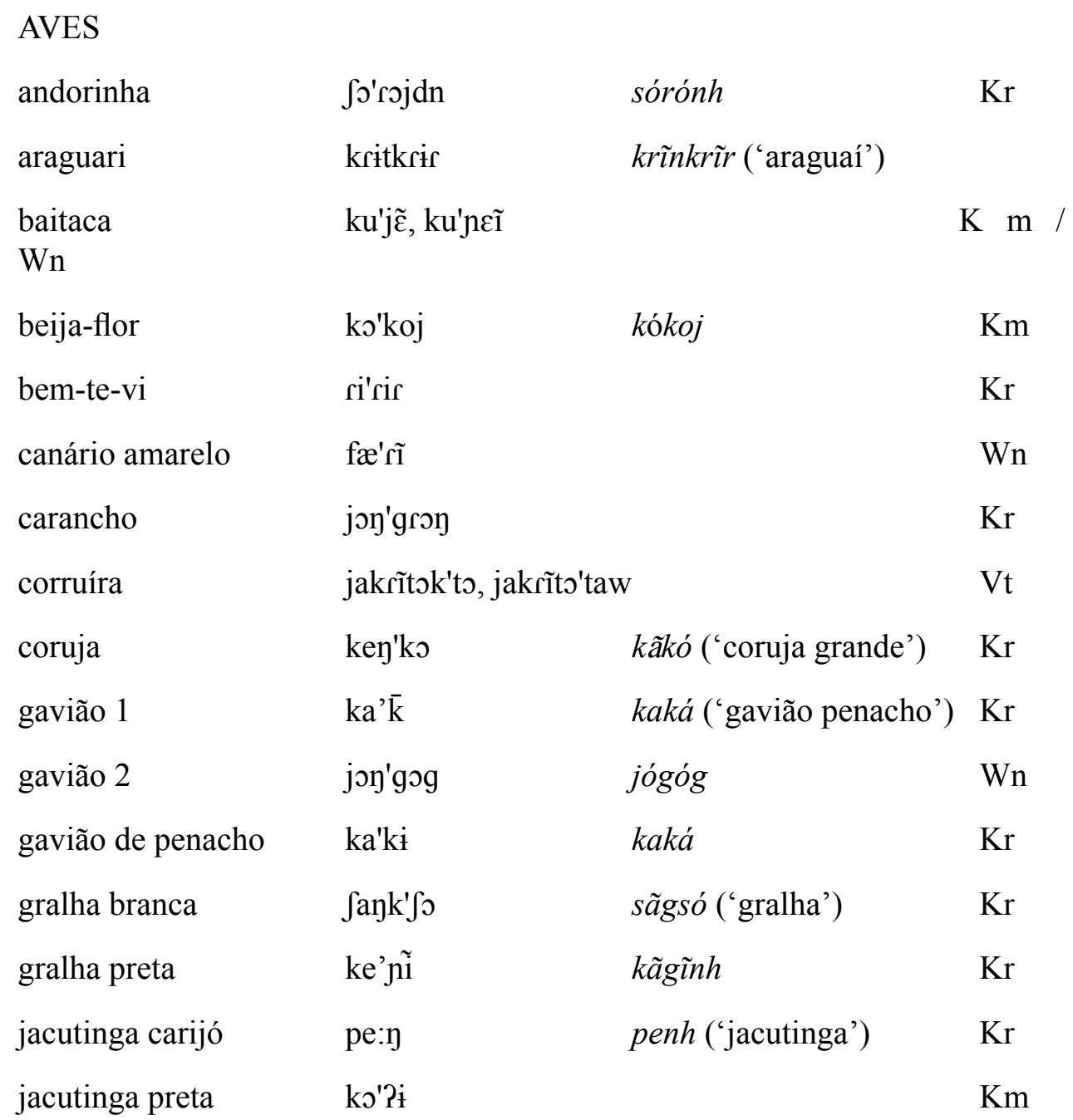




\begin{tabular}{|c|c|c|c|}
\hline macuco & vo: & vo & $\mathrm{Km}$ \\
\hline maracanã & ken'er & kẽnkẽr & $\mathrm{Kr}$ \\
\hline marreco dágua & kuj'hen & kunhhẽg & $\mathrm{Wn} / \mathrm{Vt}$ \\
\hline martim(-pescador) & krẽ'kre & & $\mathrm{Vt}$ \\
\hline nambn & $\mathrm{nd} \varepsilon$ & & $\mathrm{Kr}$ \\
\hline papagaio do peito roxo & jogĩ'o & jógjó ('papagaio’) & $\mathrm{Km}$ \\
\hline pato & goj'tn & & $\mathrm{Vt}$ \\
\hline pedreiro & 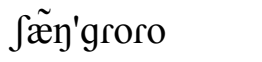 & & $\mathrm{Kr}$ \\
\hline periquito & ka'joj & & $\mathrm{Kr}$ \\
\hline picapau & 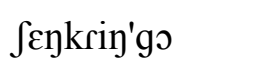 & sãkrĩgó & $\mathrm{Kr}$ \\
\hline \multicolumn{3}{|c|}{ picapauzinho pequititinho jawajאo'je } & $\mathrm{Kr}$ \\
\hline pomba bem preta & b̆tojto'ho & & $\mathrm{Kr}$ \\
\hline pomba preta & pentku'ĩ & pẽnkuĩ & $\mathrm{Kr}$ \\
\hline pomba rola & $\int \rho^{\prime} \kappa \tilde{\varepsilon}$ & sórãg ('pomba') & $\mathrm{Km}$ \\
\hline querequerê & rej're & & $\mathrm{Kr}$ \\
\hline sabiá de peito amarelo & kokridn'joj & & $\mathrm{Km}$ \\
\hline sabiá de peito branco & ygo'dnwe & gónvã & $\mathrm{Kr}$ \\
\hline sangue de boi & dzojdn & & $\mathrm{Wn}$ \\
\hline saracura & pænt'fw̄y & pẽnfág & $\mathrm{Kr}$ \\
\hline socó & $\int \mathrm{p}^{\prime} \int \mathrm{w}$ & & $\mathrm{Wn}$ \\
\hline surucuá & tuk'tu & & $\mathrm{Km}$ \\
\hline \multirow[t]{2}{*}{ tesoureiro de peito alvo } & ke'vin & kãvig & \\
\hline & & ('pássaro penacho') & $\mathrm{Kr}$ \\
\hline tesoureiro preto & $\int \tilde{1}^{\prime} \int \tilde{1}$ & & $\mathrm{Wn}$ \\
\hline tiriva (periquito) & ko'jojdn & & $\mathrm{Kr}$ \\
\hline tucano & yro: & $g r \tilde{u}$ & $\mathrm{Km}$ \\
\hline
\end{tabular}




$\begin{array}{llc}\text { tucano pequeno } & \text { yro'yro } & \mathrm{Km} \\ \text { tuiutuiú } & \text { krit'krij } & \mathrm{Kr} \\ \text { uru } & \text { pit'pir } & \text { pynpyr } \\ \text { urutau } & \text { hoj'hoj } & \mathrm{Vt}\end{array}$

\section{RÉPTEIS}

\begin{tabular}{|c|c|c|}
\hline cágado & pe'ni & pèñ̃ \\
\hline caninana, cobra d'água & $\overline{\text { pnt'ho }}$ & pỹn tĩ hó \\
\hline caninanuçu & p̄no'ror & \\
\hline cascavel & $\overline{\int " \bar{J}}$ & $s \tilde{a} s \tilde{a}$ \\
\hline coral, boicorá & bi'juj(d) & (pỹnfifi) \\
\hline jararaca & $\overline{\mathrm{p}} \mathrm{n}^{\prime} \mathrm{p} \varepsilon$ & $p \tilde{y} n p \tilde{\varepsilon}$ \\
\hline jararacuçu & $\bar{p} n$ ju'dut ku'pri & (pỹn my junjun kupri \\
\hline & & cascavelzinho) \\
\hline lagarto & jãj'ggre & jãgré \\
\hline lagarto do papo amarelo & jemu'je & jẽmũje \\
\hline rã & kõtõ & \\
\hline sapo & p $\varepsilon^{\prime}$ po & pépo \\
\hline sucuri & $\overline{\mathrm{n} n}$ 'mo & (nẽnmã 'urutu') \\
\hline urutu & fi'fi & \\
\hline
\end{tabular}

\section{PEIXES}

\begin{tabular}{|c|c|c|}
\hline dourado & pirã'ju & \\
\hline lambari & kræygufw̄̄ $\varsigma^{\prime} \sqrt{1}$ & [krẽkofár sĩ] \\
\hline peixe & kræyku'fw̄r & $\begin{array}{l}\text { krẽkofár } \\
\text { ('peixe pequeno') }\end{array}$ \\
\hline
\end{tabular}




\section{INSETOS}

\begin{tabular}{|c|c|c|}
\hline abelha & $\mathrm{m} \wedge \mathrm{y}, \mathrm{m} \wedge \mathrm{y} \overline{\mathrm{b} g}$ & (mỹg 'mel') \\
\hline aranha & Ju:'krin & sukrig \\
\hline barata & jan'to & \\
\hline besouro & $\tilde{j}$ fa kun'did & (jã $f a$ 'fezes') \\
\hline \multirow[t]{2}{*}{ bicho cabeludo } & taj'ygoku'fõ & ('mandorová' + kusũg \\
\hline & & 'vermelho') \\
\hline butuca & pen'tu & pãtu \\
\hline caramujo & dur & nun \\
\hline coró de palmeira & fæn'dju & \\
\hline formiga & pæt'kring & pénkrig \\
\hline gafanhoto & o'p:a: & ópã \\
\hline grilo & op $\varepsilon^{\prime} \int \mathrm{i}$ & (firég, kriggrig, kruj) \\
\hline lesma & pa:r & pãr ('lesma preta”) \\
\hline mandorová & taj'ygo & tãnh gó ('manduruva') \\
\hline minhoca & jo'kin & $\begin{array}{l}\text { jókynh ('larva nas } \\
\text { folhas caídas') }\end{array}$ \\
\hline mosca & $\mathrm{ka}^{r} \mathrm{tj}$ & ka tánh \\
\hline mosquito miúdo & $\mathrm{ka}^{\prime} \sqrt{1}$ & \\
\hline mosquito que morde & $\mathrm{ka}$ & ka ('mosquito') \\
\hline pernilongo & $\int \mathrm{i}$ & \\
\hline pulga & kam'po & kãpó \\
\hline vaga-lume & ke'nĩ & kynin \\
\hline vespa & kok ${ }^{\mathrm{u}}$ fo & kógfo \\
\hline
\end{tabular}

